\title{
Inheritance of human longevity in Iceland
}

\author{
Hjalti Gudmundsson ${ }^{1}$, Daniel F Gudbjartsson ${ }^{1,3}$, Augustine Kong ${ }^{1,2}$, \\ Hákon Gudbjartsson ${ }^{1}$, Mike Frigge ${ }^{1}$, Jeffrey R Gulcher ${ }^{1}$ and Kári Stefánsson ${ }^{1}$
}

${ }^{1}$ Decode Genetics Inc, Reykjavik, Iceland; ${ }^{2}$ Departments of Human Genetics and Statistics, University of Chicago, Chicago, IL; ${ }^{3}$ Institute of Statistics and Decision Sciences, Duke University, Durham, NC, USA

The idea that human longevity is influenced by genetic factors has recently received strong support from work on other species. On the basis of partial population studies and selected kinships, significant correlations between the ages of parents and offspring have been reported, and some but not all twin studies have confirmed that human longevity is moderately inherited. However, studies based upon a relatively small proportion of a population are susceptible to sampling error and selection bias. Here we report the use of a comprehensive population-based computerised genealogy database to examine multigenerational relationships among those who live to the 95th percentile in Iceland. We have developed a clustering tool which can generate large extended pedigrees connecting individuals from any list using the genealogy database. First degree relatives of those living to the 95th percentile are almost twice as likely to live to the 95th percentile compared with controls. Furthermore, we have developed an algorithm which we have named the Minimum Founder Test (MFT) to examine the degree of relatedness of any population-based list of individuals to estimate whether a trait has a familial component. The data indicate that there is a significant genetic component to longevity. In addition, age-specific death rates are significantly lower in the offspring of long-lived parents compared with controls, especially after age 70. European Journal of Human Genetics (2000) 8, 743-749.

Keywords: human longevity; genetics; genealogy; inheritance; Iceland; relative risk

\section{Introduction}

Whilst old age has been attained by a larger proportion of recent generations throughout the world than by previous generations, convincing evidence supporting a role for genetics in longevity has eluded scientists for a number of reasons. In 1934 Pearl and Pearl ${ }^{1}$ found a positive correlation between the life spans of individuals who lived 90 years or more and the life spans of their parents and grandparents. Abbott et $\mathrm{al}^{2}$ showed that long-lived parents tended to have long-lived children. Gavrilova et $\mathrm{al}^{3}$ found that life spans of parents and offspring correlate, especially the longest life spans in European aristocratic families. Several twin studies estimated that longevity is inherited, ${ }^{4-6}$ although in one recent twin study the genetic component was less clear. ${ }^{7}$ The life span of Jeanne Calment who recently died in France at 122 years of age is the longest ever recorded in a human; ${ }^{8,9}$ a

Correspondence: Jeffrey R Gulcher or Kári Stefánsson.

E-mail: jgulcher@decode.is, kstefans@decode.is

Received 24 December 1999; revised 17 May 2000; accepted 7 June

2000 subsequent study of her ancestors revealed that her relatives lived longer than in the control families. However, many of these studies are based on selected pedigrees or a relatively small segment of the population which may lead to selection or socio-economic bias.

Iceland is an ideal nation for genetic studies in general, because it has an isolated population with extensive genealogical records spanning its 11 centuries of history. ${ }^{10,11}$ At deCODE genetics a computerised genealogy database has been developed which uses, as its raw data, the extensive genealogical information collected over the last 10 centuries by the government-supported churches and public institutions. It includes all 270000 living Icelanders in addition to most of their ancestors since the time of the settlement in the ninth century. Over 600000 are currently registered in the database; it has been estimated that around 1 million Icelanders have lived since the country was settled.

Iceland is also an ideal country for an epidemiological study of the contribution of genetic factors to longevity. First, the mean life expectancy of Icelanders is among the highest 
in the world, currently 81.3 years for females and 76.4 years for males. ${ }^{12}$ In this century, the probability of Icelanders reaching 90 years of age is about $12 \%$ of all live births. Second, population-based lists of almost all Icelanders who have lived over any given age within the last few centuries are obtainable. The genealogy database that we have developed contains both birth date and death date allowing for systematic determination of individual and population life spans. A population-based ascertainment of any trait is an enormous advantage since it decreases the possibility of sampling bias. Instead of relying on the records of selected families of the aristocracy (the raw material for many previous studies of human longevity), we can sample a crosssection of an entire society. Third, we can more carefully control for socio-economic influences on longevity. Some have shown that longevity may co-segregate in certain families on the basis of socio-economic status al one. ${ }^{13,14}$ This bias may be most evident in studies of longevity that are based on nuclear families covering only a couple of generations. In contrast, our study is a population-based examination of relationships extending beyond nuclear families, using the genealogy database. Iceland was, until recently, a relatively poor country with a smaller gap between the wealthiest and the poorest in society compared with most other European societies. For this reason, along with the fact that housing was uniformly primitive for many centuries, the upper and lower classes differed little culturally. ${ }^{15}$ Furthermore, Iceland has had a single payer healthcare system with universal access for the past 80 years, thus diminishing another potential basis of bias.

We have taken advantage of our population-based genealogical database, which can be efficiently interrogated to determine relationships among any set of Icelandic individuals. We can use this tool to search for genetic components influencing any given trait in a way that may be less biased than the traditional word-of-mouth method of pedigree ascertainment. This is a population-based study that reaches all living Icelanders and the majority of their ancestors and is, therefore, relatively free of selection bias. Using the years of birth and death in the database, we have defined groups of individuals within the same age range at death.

We have developed a clustering function that takes a list of individuals and determines how many on the list are related to any given individual and by what meiotic distance, using the genealogy database. These clusters can then be displayed using pedigree drawing software. It is possible that large extended pedigrees containing multiple long-lived individuals found using this novel method may represent unusual cases of extensive familial clustering, not representing the general relationship pattern among long-lived individuals. Therefore we also measured the relatedness of the individuals within the entire long-lived group in several different ways.

In order to determine the significance of clustering of any group using the computerised genealogy database, we developed an algorithm which we call the Minimum Founder Test
(MFT). This has allowed us to address the following questions. Is the degree of clustering of long-lived individuals elucidated by our clustering function more than would be found when taking the same number of control individuals and applying the clustering function? Or should one expect a great deal of clustering among individual s on any list, since Iceland was founded by a limited number of immigrants who have expanded in isolation ever since. The MFT determines the minimum number of founders a certain number of generations back in time required to account for the test group and compares it to the minimum number of founders required to account for a control group of similar size.

\section{Materials and methods Genealogy database and cluster function}

Our laboratory has a current genealogy project which involves electronic registration of all available genealogy for the last ten centuries in Iceland including genealogy manuscripts, censuses, church records and telephone books. The genealogy database is stored and maintained within a relational database. Each record in the database consists of a personal identifier (PID), identifier of parents (FID and MID), gender, dates of birth and death. We have shown by examining the mitochondrial sequences of maternally related individuals that the database connection accuracy exceeds $99.3 \% .^{16}$ Based on genotyping over 15000 Icelanders, we estimate that the sum of laboratory error rate and non-paternity rate is less than $1.5 \%$. Each version of the computerised geneal ogy database is reversibly encrypted by the Data Protection Commission of Iceland before arriving at the laboratory (http://www.decode.is/ppt/protection/ index.htm). All algorithms that work on family relations were implemented outside the database, in memory-based programs, since relational databases are not well suited to dynamic programming algorithms. We have developed recursive pedigree algorithms that find all ancestors in the database who are related to each member of the input list within a given number of generations back. Using these groups the cluster function then searches for ancestors who are common to any two or more members of the input lists. These pedigrees are then drawn by the Cyrillic pedigree drawing program (Cherwell Scientific Publishing Ltd).

\section{Minimum Founder Test}

The MFT compares the lowest number of ancestors whose descendents include a particular set of affected individuals with an empirical distribution of the lowest number of ancestors for sets of matched controls. Let $S$ be a set of individuals. We say $A$ is an ancestor set of $S$ if $S$ is a subset of the set comprised of $A$ and all the descendents of $A$. In other words, each person in $\mathrm{S}$ has at least one ancestor, possibly himself or herself, in the set A. S itself is a simple example of one such set $A$. The test statistic we use, $F(S, Y)$, is the minimum size of all ancestor sets of $S$ such that the ancestors 
are born not earlier than year $Y$. Clearly, $F(S, Y)$ is nonincreasing as $Y$ decreases. Five hundred random control sets (equivalent in size to the affected set) for producing the empirical distribution are selected from the geneal ogy such that they each have the same size and year of birth distribution as the affected set. We assess the significance of the result (ie whether the affected individuals have significantly fewer ancestors than randomly selected sets of individuals) in two ways. First, we deliver a graph displaying the statistic, as a function of $Y$, for the affecteds along with the mean and +/- two standard deviations of the relevant control distributions. We also report how far $F(S, Y)$ for the affecteds is from the control mean in standard deviation units.

There exists a computational problem in determining $F(S, Y)$. Only in specialised cases is it possible to compute this exactly. This problem of finding the fewest number of ancestors for a set of individuals can be reduced to a setcovering problem which has an extensive literature in mathematics and computer science. ${ }^{17}$ We have implemented a branch and bound algorithm with occasional greedy steps ${ }^{17}$ in order to solve this computational problem.

\section{Survival analysis}

We used logistic regression to model discrete death rates according to Efron. ${ }^{18}$ We calculated death rates for individuals born between 1870 and 1900 and living older than 30, putting ages and death rates into 5 -year bins. We fitted a quadratic model to the death rate data with a constant shift on the logit scale $(\operatorname{logit}(P)=\log (P /(1-P)))$ for individuals with long-lived parents, using the standard logistic package of S-plus (StatSci). The fit was quite good, and a non-parametric death rate curve (having a separate death rate for every bin) did not significantly improve the fit. Using different first and second order terms for individuals with long-lived parents also did not improve the fit significantly.

\section{Kinship coefficients}

We developed a type of kinship coefficient (KC) in order to assess whether identical-by-descent (IBD) sharing among a set of affected individuals is greater than that for sets of matched controls. The KC for a pair of individuals is the probability that, for a particular autosomal locus, two randomly selected alleles, one from each individual, are IBD. ${ }^{19}$ We determined the average pairwise KC for the set of affected individuals and compared this with the distribution of the average pairwise KC for sets of matched controls. $M$ atched control sets were developed as described for use in the MFT. Specifically, to evaluate statistical significance, we generated 1000 'sets' of controls and cal culated the average pairwise kinship coefficient for each set of controls. The empirical $P$ value is determined by comparing the average pairwise kinship coefficient of the affecteds with the 1000 average pairwise kinship coefficients of the 1000 control sets. This is similar to the method used to assess cancer risk in
Utah Mormon genealogies. ${ }^{20}$ When no control values are as or more extreme than for the affecteds, we report the $P$ value as $<0.001$.

Due to the size of the pedigrees there is a computational problem in determining the $\mathrm{KC}$ exactly. We used Monte Carlo simulations to approximate the average pairwise KC. For each set of individuals $\mathrm{S}$ we determined the entire set of founders going back 15 generations. Each founder was given a set of unique alleles. Thus, for $F$ founders, there are $2 F$ unique alleles in the founder set. Mendelian inheritance laws were used to simulate transmissions of alleles to offspring throughout the pedigree. Then for each pair of individuals in $S$ the fraction of IBD alleles was determined. We ensured that the Monte Carlo errors have a negligible effect on the reported results.

\section{Results}

We first produced pedigrees for those who have lived to at least 90 years and were born between 1870 and 1900 . Figure 1 depicts one of many large extended pedigrees which included numerous individuals who reached the age of 90 and beyond.

In order to control for the background relatedness in Iceland and to examine the relatedness of an entire group of long-lived individuals we applied the MFT. From the genealogy database a more extreme phenotype was identified as those living to the 95th percentile in age and born between 1870 and 1900. All percentiles were adjusted for year of birth and sex. This yielded a list of 1531 individuals including 770 females over 96 and 761 males over 94 years. Figure 2 shows the minimum number of founders vs year of birth of the founders. Note that within the current generation each long-lived individual was a founder to himself or herself; thus there were 1531 founders. The figure shows that the minimum number of founders continued to decrease with the number of previous generations until it levelled off to about 600 in the year 1500 (about 39\% of the test list). We performed the test on 500 lists of 1531 random controls each (irrespective of age at death), in which each individual in the experimental group of long-lived individuals was matched to an individual born in the same year. This produced a distribution of control curves that could be compared with the long-lived curve to determine its significance. The longlived group was clearly different from the control groups and fell more than two standard deviations from the mean throughout the past 500 years, reaching a maximum of 8.2 standard deviations around the year 1820. This indicates that the members of the long-lived group have descended from fewer founders born in each of these years. Therefore, they are more related to each other than the control group.

In addition, we explored the age-specific death rates of the offspring of long-lived individuals (95th percentile) born between 1870 and 1900 and compared them with the death rates of the offspring of control populations born within the 
same period. As shown in Figure 3 the death rates of offspring of long-lived individuals were generally lower than those of offspring of controls, particularly after the age of 70 . The shift in death rate above the age of 70 was significant for all the groups $(P<0.02$ for female offspring of long-lived fathers, $P$ about $10^{-4}$ for the other groups). The difference in death rates began as early as 50 years of age in the male offspring of long- lived fathers $(P<0.01)$. It is notable that the shift in the death rate curves of male offspring of long-lived parents was independent of whether the older parent was the mother or the father (Figure 3a). The shifts in probability for male and female offspring of long-lived mothers and the male offspring of long-lived fathers were roughly 0.3 on the logit scale, which for ages 70-90 corresponded to those offspring

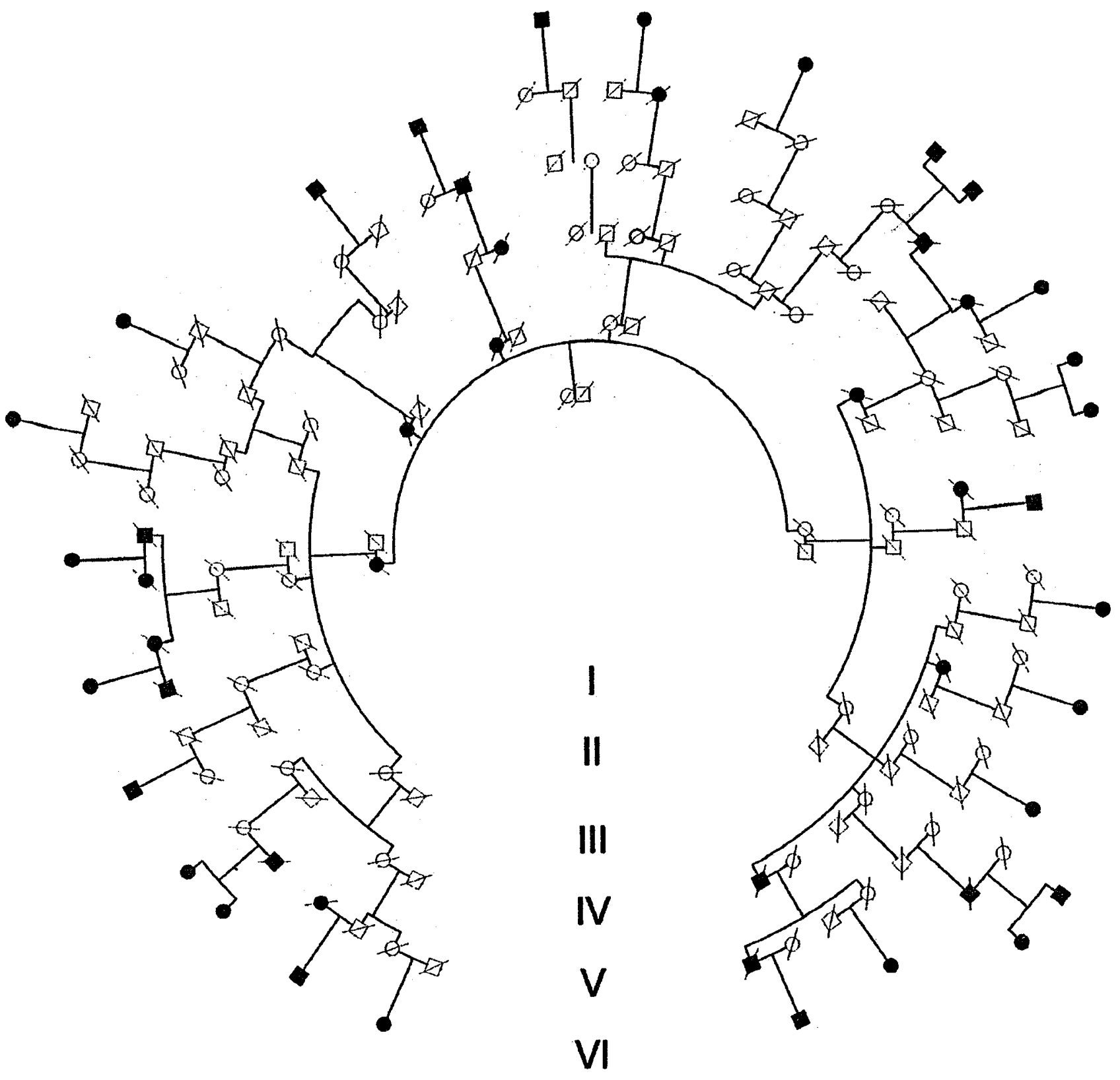

VII

Figure 1 This is an example of a pedigree, which includes a large number of individuals living more than 90 years (filled circles and squares). The pedigree reaches six generations back to the year 1730 . Not all siblings or relatives are shown, but the average number of siblings of the 27 long-lived individuals still living is about five. 
with a $25 \%$ lower age-specific death ratethan the offspring of controls. The female offspring of long-lived fathers had a shift of 0.15 , corresponding to a $14 \%$ decrease in death rate for ages 70-90. These values can be compared with those for husbands and wives where any significant shift in death rate is assumed to be principally due to environmental effects. The wives of long-lived husbands had a shift of 0.10 , whereas the shift was 0.15 for the husbands of long-lived wives. The $P$ values for these shifts are 0.11 and 0.04 , respectively. Although these values are not very significant, we do not believe that there is no environmental component to longevity, only that the effect is small and difficult to estimate with precision from these data.

Wealso used more conventional tests of relatedness among our long-lived individuals. Table 1 shows the calculated risk ratio, $\lambda$, which is the rate of longevity for given relatedness of long-lived (95th percentile) probands divided by the prevalence of longevity in the general population. The risk ratios for sibs and parents were similar, 1.8 and 1.7, respectively, while the risk ratios for grandparents-grandchildren and cousin-cousin pairs decreased to 1.4 and 1.3 , respectively. ${ }^{21}$ This rate of decrease of the relative risk with meiotic distance (the relative risk minus one, halves with each meiotic step between the compared long-lived relatives) is more consistent with one or a few genes contributing to longevity than simply an avoidance of a high number of death causing genes. ${ }^{21}$ However, it should be mentioned that the data are consistent with an additive multilocus model (multiple genes

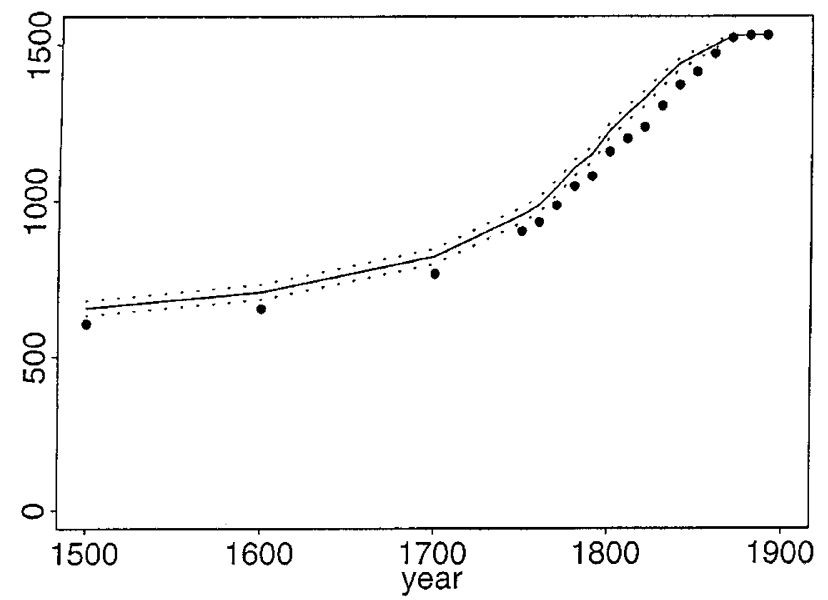

Figure 2 The Minimum Founder Test demonstrates a significant difference in the minimum number of common ancestors of a group of 1531 long-lived individuals who lived to the 95th percentile in age in comparison with control groups of individuals matched to the long-lived group according to year of birth and sex. Each curve shows the minimum number of founders as a function of the birth years of the founders. The large solid dots represent the group whose members live to the 95th percentile age; the solid line depicts the mean for 500 control groups and the dotted lines represent two standard deviations from the mean. working independently, ie locus heterogeneity ${ }^{21}$ ). As another measure of relatedness, kinship coefficients were determined. ${ }^{19}$ The kinship coefficient of the long-lived group (95th percentile) was $1.0 \times 10^{-4}$ compared with control groups which gave a kinship coefficient of $0.83 \times 10^{-4}$ (standard deviation of $2.93 \times 10^{-6}, \quad \mathrm{P}<0.001$ ). There

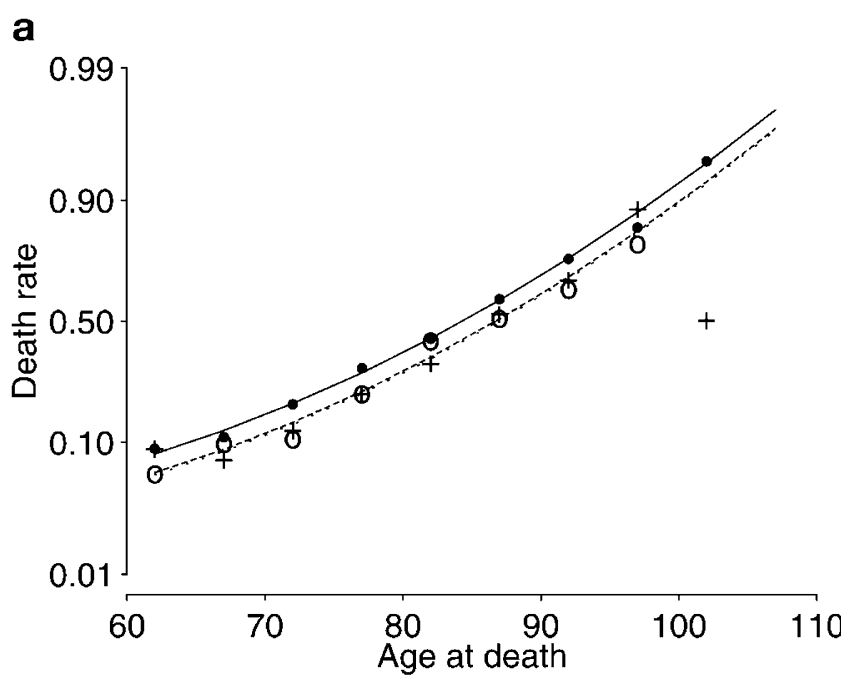

b

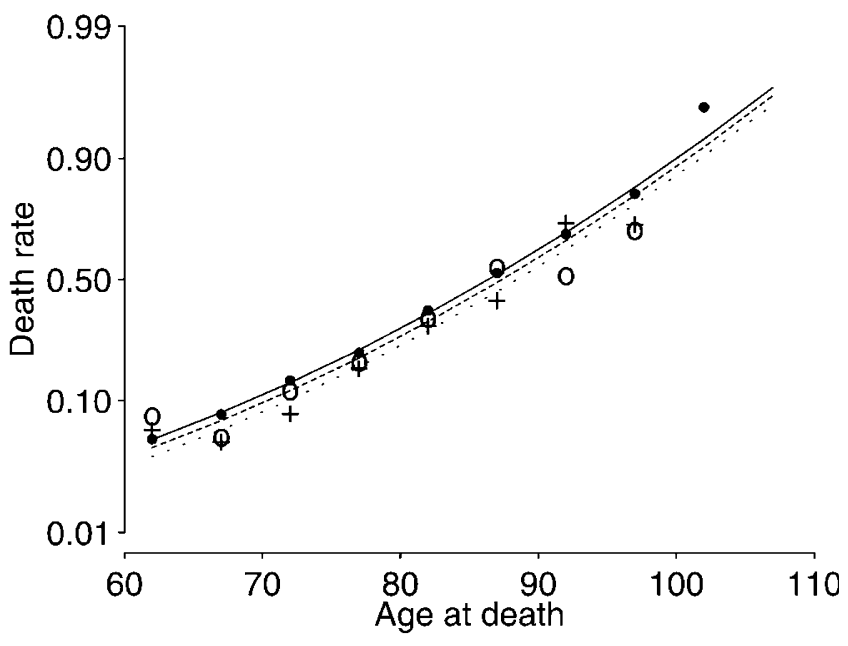

Figure 3 The age-specific death rate (hazard) curves show that the probability of dying within the next five years, given that a person lives to the age on the $x$-axis, depends on the age of death of the parents. ${ }^{18}$ The $y$-axis is the death rate on a logit scale. The dots show the observed death rate of offspring of controls and the solid lines show the quadratic fit through these points. The crosses and the dotted line similarly represent offspring of long-lived mothers and the 0 s and the dashed line for offspring of long-lived fathers. Figure $3 a$ represents male offspring and Figure $3 b$ represents female offspring. The death rates of offspring of long-lived parents are significantly lower than those of offspring of controls over the age of 70 . 
remained a significant difference between kinship coefficients even after first degree relatives were removed.

\section{Discussion}

Our results suggest a clear familial component in longevity. This can result from either a genetic component or environmental factors shared by family members. Previous studies have shown a correlation between longevity and socioeconomic status, profession, education and health care. ${ }^{13,14}$ In studies of longevity based on nuclear families, the familial aggregation of longevity may be related to socio-economic status which itself can show familial aggregation. The impact of socio-economic bias on this study is unlikely since we have shown that the familial component in longevity extends for multiple generations beyond the nuclear family. It has been noted that studies of relatives beyond the nuclear family decrease the chance that observed associations are related to shared environmental factors. ${ }^{22}$ In addition, long-lived individuals remain more closely related to each other than controls even when first degree relatives are removed. Finally, we have found only a small correlation of death rates between spouses, suggesting that the genetic component found even within families exceeds the environmental component. Historically, Iceland has been relatively uniform with respect to cultural and socio-economic status compared with most other countries, with little difference between the wealthiest and poorest families, further decreasing the likelihood of bias. Furthermore, the generations examined in this study span an era when there was significant reshuffling of the wealthiest and poorest families in Iceland. ${ }^{23}$ Therefore, our data specifically suggests a genetic component in longevity.

Recently, Westendorp and Kirkwood ${ }^{24}$ published a study showing a relationship between longevity and reproductive success among the British aristocracy. Using selected pedigrees of the British royal family, they showed a negative correlation between female longevity and the number of

\section{Table 1}

\begin{tabular}{lll}
\hline & $\lambda_{\mathrm{R}}$ & $\lambda_{\mathrm{R}}-1$ \\
\hline sibs & 1.8 & 0.8 \\
parent/offspring & 1.7 & 0.7 \\
grandparent/grandchild & 1.4 & 0.4 \\
uncle/nephew & 1.4 & 0.4 \\
cousin/cousin & 1.3 & 0.3
\end{tabular}

Table 1 shows the risk ratio, $\lambda_{R}$, for various relationships. ${ }^{19}$ For example, given two sibs, $\lambda$ (sibs) is the probability that the second sib is long-lived (95th percentile of age) given that the first one is long-lived, divided by the probability that the second sib is longlived without any information about the first sib (the population frequency of longevity). The $\lambda_{R}-1$ declines by a factor of about 2 for each meiotic step between the long-lived relative pairs which is consistent with one major gene (or more than one in an additive multilocus model) influencing human longevity in the Icelandic population. $^{21}$ their progeny, and a positive correlation between the longevity of women and the age at which they gave birth to their first child. We have carried out a similar study on our much larger population within the same era and have not found any evidence that human longevity occurs at the cost of reproductive success (unpublished observation).

The genetic component of longevity could be explained either by the absence of disease genes, or by the presence of protective 'Iongevity' genes that counteract aging. Several genes have shown an association with longevity in human populations such as apolipoprotein $\mathrm{E}$, angiotensin converting enzyme, HLA-DR, and lipoprotein(a). ${ }^{25-29}$ There was also a reported association with human mitochondrial genotypes. ${ }^{30}$ Furthermore, it has been suggested that telomere metabolism may play a role. ${ }^{31}$ Recent work in both $C$. elegans and Drosophila demonstrate that specific alterations in a single gene can dramatically extend life span. ${ }^{32-38}$ These genes appear to be involved in signalling pathways that may regulate cell metabolism. Interestingly, there is evidence that the daf- 2 gene which extends life in $\mathrm{C}$. elegans, works systemically. ${ }^{38}$

Longevity is a complex phenomenon and the result of numerous interacting factors including genetic, environmental and behavioural components. ${ }^{14,39-41}$ Some events that may be important could take place long before the old age of the individual. In our population-based study, however, we demonstrated a genetic component to human longevity that appears to influence death rates most dramatically after the age of 70 . Our study demonstrates a monotonic fall in the relative risk ratios with meiotic distance consistent with one or a few major genes independently influencing human longevity (additive multilocus model), at least in the Icelandic population. ${ }^{21}$ In addition, the significance in genealogic clustering of patients extends many generations back, well beyond the nuclear families, suggesting that human life span is not simply the result of fortuitous dodging of numerous genetic and environmental bullets.

In conclusion, we have clearly shown that there is a strong familial component to longevity. Second, it is likely that this familial component is a genetic one. Third, this genetic component to longevity may depend on one or a few genes.

\section{Acknowledgements}

Hjalti Gudmundsson and Daniel F Gudbjartsson contributed equally to this work.

\section{References}

1 Pearl R, Pearl RW: The Ancestry of the Long-lived. Johns Hopkins University Press: Baltimore, 1934.

2 Abbott $\mathrm{MH}$, Murphy EA, Bolling DR, Abbey $\mathrm{H}$ : The familial component in longevity. A study of offspring of nonagenarians. II. Preliminary analysis of the completed study. Johns Hopkins M ed J 1974; 134: 1-16. 
3 Gavrilova NS, Gavrilov LA, Evdokushkina GN et al: Evolution, mutations, and human longevity: European royal and noble families. Hum Biol 1998; 70: 799-804.

4 Jarvik LF, Falek A, Kallmann FJ, Lorge I: Survival trends in a senescent twin population. Am J Hum Genet 1960; 12: 170-179.

5 Wyshak G: Fertility and longevity in twins, sibs, and parents of twins. Soc Biol 1978; 25: 315-330.

6 Herskind AM, McGue M, Holm NV, Sorensen TI, Harvald B, Vaupel JW: The heritability of human Iongevity: a populationbased study of 2872 Danish twin pairs born 1870-1900. Hum Genet 1996; 97: 319-323.

7 Ljungquist B, Berg S, Lanke J, McClearn GE, Pedersen NL: The effect of genetic factors for longevity: a comparison of identical and fraternal twins in the Swedish Twin Registry. J Gerontol A Biol Sci Med Sci 1998; 53: M441-M446.

8 Robine JM, Allard M: The oldest human. Science 1998; 279: 1834-1835

9 Schachter F: Causes, effects, and constraints in the genetics of human longevity. Am J Hum Genet 1998; 62: 1008-1014.

10 Gulcher J, Stefansson K: Population genomics: Iaying the groundwork for genetic disease modeling and targeting. Clin Chem Lab Med 1998; 36: 523-527.

11 Gulcher J, Stefannson K: The Icelandic Healthcare Database: A Tool to Create Knowledge, a Social Debate, a Bioethical and Privacy Challenge. Medscape (http://molecularmedicine.medscape.com/ 10835.rhtml), 1999.

12 Statistics Iceland. 1998. http://www.hagstofa.is

13 Vagero D, Lundberg O: Socio-economic mortality differentials among adults in Sweden. In: Lopez A, Caselli G, Valkonen T (eds). Adult Mortality in Developed Countries. Oxford, 1995.

14 Christensen K, Vaupel JW: Determinants of Iongevity: genetic, environmental and medical factors. J Intern Med 1996; 240: 333-341.

15 Gunnarsson G: Einokunarverslun og íslenskt samfélag 1602-1787. Upp er bołið Ísland. Örn og Örlygur: Reykjavik, 1987.

16 Sigurłardóttir S, Helgason A, Gulcher JR, Stefansson K, Donnelly $P$ : The mutation rate in the human mtDNA control region. Am J Hum Genet 2000; 66: 1599-1609.

17 Cormen TH, Leiserson CE, Rivest RL: Introduction to Algorithms. MIT Press: Cambridge, MA, 1990.

18 Efron B: Logistic regression, survival analysis, and KaplanMeier curve. J Amer Stat Assoc 1988; 83: 414-425

19 Lange K: Mathematical and Statistical Methods for Genetic Analysis. Springer: New York, 1997.

20 Skolnick M, Bishop DT, Carmelli E et al: A population-based assessment of familial cancer risk in Utah Mormon genealogies. In: Arrighi FE, Rao PN, Stubblefield E (eds). Genes, Chromosomes, and Neoplasia. Raven Press: New York, 1981.

21 Risch N: Linkage strategies for genetically complex traits. I. Multilocus models. Am J Hum Genet 1990; 46: 222-228.

22 Sadovnick AD, Macleod PMJ: The familial nature of multiplex sclerosis: empiric recurrence risks for first, second, and thirddegree relatives of patients. Neurology 1981; 31: 1039-1041.

23 Gunnarsson G: Afkoma og afkomendur meiri háttar fólks 1550-1800. Íslenska sögubingið 1997; 2: 118-132.
24 Westendorp RG, Kirkwood TB: Human Iongevity at the cost of reproductive success. Nature 1998; 396: 743-746.

25 Schachter F, Faure-Delanef L, Guenot F et al: Genetic associations with human Iongevity at the APOE and ACE Ioci. Nat Genet 1994; 6: 29-32.

26 Louhija J, Miettinen HK, Kontula K, Tikkanen MJ, Miettinen TA, Tilvis RS: Aging and genetic variation of plasma apolipoproteins. Relative loss of the apolipoprotein E4 phenotype in centenarians. Arterioscler Thromb 1994; 14: 1084-1089.

27 Takata H, Suzuki M, Ishii T, Sekiguchi S, Iri H: Influence of major histocompatibility complex region genes on human longevity among Okinawan-Japanese centenarians and nonagenarians. Lancet 1987; 2: 824-826.

28 I vanova R, Henon N, Lepage V, Charron D, Vicaut E, Schachter F: HLA-DR alleles display sex-dependent effects on survival and discriminate between individual and familial Iongevity. Hum Mol Genet 1998; 7: 187-194.

29 Thillet J, Doucet C, Chapman J, Herbeth B, Cohen D, Faure Delanef L: Elevated lipoprotein(a) levels and small apo(a) isoforms are compatible with Iongevity: evidence from a large population of French centenarians. Atherosclerosis 1998; 136 389-394.

30 Tanaka M, Gong JS, Zhang J, Yoneda M, Yagi K: Mitochondrial genotype associated with Iongevity. Lancet 1998; 351 185-186.

31 Morin GB: Telomere control of replicative lifespan. Exp Gerontol 1997; 32: 375-382.

32 Friedman DB, Johnson TE: A mutation in the age-1 gene in Caenorhabditis el egans lengthens life and reduces hermaphrodite fertility. Genetics 1998; 118: 75-86.

33 Kimura KD, Tiessenbaum HA, Liu Y, Ruvkun G: daf-2, an insulin receptor-like gene that regulates longevity and diapause in Caenorhabditis elegans. Science 1997; 277: 942-946.

34 Lin K, Dorman JB, Rodan A, Kenyon C: daf-16: an HNF3/forkhead family member that can function to double thelifespan of Caenorhabditis el egans. Science 1997; 278: 1319-1322.

35 Ewbank JJ, Barnes TM, Lakowski B, Lussier M, Bussey H, Hekimi S: Structural and functional conservation of the Caenorhabditis elegans timing gene clk-1. Science 1997; 275: 980-983.

36 Lin YJ, Seroude L, Benzer S: Extended life-span and stress resistance in the Drosophila mutant methuselah. Science 1998 282: 943-946.

37 Ogg S, Paradis S, Gottlieb S et al: The Fork head transcription factor DAF-16 transduces insulin-like metabolic and Iongevity signals in C. elegans. Nature 1997; 389: 994-999.

38 Apfeld J, Kenyon C: Cell nonautonomy of C. elegans daf-2 function in the regulation of diapause and life span. Cell 1998 95: 199-210.

39 Finch CE, Tanzi RE: Genetics of aging. Science 1997; 278: 407-141.

40 Finch CE: Longevity, Senescence and the Genome. The University of Chicago Press: Chicago, 1993.

41 Schachter F, Cohen D, Kirkwood T: Prospects for the genetics of human Iongevity. Hum Genet 1993; 91: 519-526. 